\section{Adaptación cultural y validación del cuestionario Ocular Surface Disease Index en una población chilena}

\author{
LEONIDAS TRAIPE ${ }^{1}$, FUAD GAURO ${ }^{1}$, MARÍA CLAUDIA GOYA ${ }^{1,3, a}$, \\ CRISTIAN CARTES ${ }^{1}$, DANIELA LÓPEZ ${ }^{1,3, a}$, DANIELA SALINAS ${ }^{1, a}$, \\ MAURICIO CABEZAS ${ }^{1}$, CLAUDIA ZAPATA ${ }^{1}$, PATRICIA FLORES ${ }^{1,2, a}$, \\ GONZALO MATUS ${ }^{1}$, CHRISTIAN SEGOVIA ${ }^{1, a}$, \\ ALBA LEÓN $^{4, b}$, REMIGIO LÓPEZ ${ }^{1,2, \mathrm{c}}$
}

\section{Validation of the Ocular Surface Disease Index Questionnaire for Chilean patients}

Background: Ocular Surface Disease Index (OSDI) is the most commonly used questionnaire worldwide to detect dry eye disease. Although it is massively used in clinical practice in Chile, its use has not been validated yet in the country. Aim: To develop a cultural adaptation and to validate the OSDI questionnaire for the Chilean population. Material and Methods: For cultural adaptation, a translation, retro-translation and an expert panel review was carried out. The resulting questionnaire was applied to a pilot group of twelve participants and their comments were considered for the final questionnaire version. The final questionnaire was applied to a non-random sample of 200 patients aged $53 \pm 17$ years ( $75 \%$ women). Internal consistency and construct validity were evaluated by Cronbach's alpha and exploratory factor analysis respectively. Results: According to the OSDI score, $81 \%$ of respondents had dry eye (55\% severe). Reliability was 0.91 and factor analysis resulted in three factors explaining $75.4 \%$ of the total variance. Conclusions: The OSDI questionnaire version obtained in this study demonstrated excellent internal consistency values and adequate construct validity making it applicable to clinical practice and dry eye research.

(Rev Med Chile 2020; 148: 187-195)

Key words: Dry Eye Syndromes; Surveys and Questionnaires.
'Unidad de Lágrima y Superficie Ocular (ULSO). Centro de la Visión (CEV) filial Clínica Las Condes. Santiago, Chile. ${ }^{2}$ Instituto de Ciencias Biomédicas (ICBM). Facultad de Medicina. Universidad de Chile. Santiago, Chile.

${ }^{3}$ Departamento de Tecnología Médica. Facultad de Medicina. Universidad de Chile. Santiago, Chile.

${ }^{4}$ Coorporación para el Apoyo de la Investigación Científica en Nutrición (INTA). Universidad de Chile. Santiago, Chile. aTecnóloga médica.

bBioestadística.

'Bioquímico.

Trabajo no recibió financiamiento. Los autores declaran no tener conflicto de interés.

Recibido el 20 de mayo de 2019, aceptado 17 de enero de 2020 .

Correspondencia a: Dr. Leonidas Traipe Itraipe@gmail.com
L

a evaluación de los síntomas de la enfermedad de la superficie ocular (ESO) ha adquirido un papel preponderante en el abordaje clínico y de investigación en torno al síndrome de ojo seco (SOS). Una proporción de pacientes sintomáticos con enfermedad leve a moderada no presentan hallazgos clínicos ${ }^{1}$, lo cual se sustenta en la falta de concordancia entre síntomas y signos reportada en la literatura ${ }^{2,3}$. En este contexto, los cuestionarios clínicos permiten estandarizar y cuantificar los síntomas, y han sido diseñados para ser respondidos en forma autónoma por los pacientes, evitando la influencia del clínico o investigador.

El reporte de Dry Eye Workshop II (DEWS II) reconoce 17 cuestionarios para evaluar el SOS, tanto en contextos clínicos como de investigación (estudios clínicos y epidemiológicos), de los cuales 12 han sido validados ${ }^{4}$. Asimismo, recomienda para la práctica clínica habitual el uso alternativo de dos tests: DEQ-5 y OSDI, este último ampliamente utilizado ${ }^{5}$. 
El Ocular Surface Disease Index (OSDI), desarrollado por The Outcome Research Group at Allergan $\operatorname{Inc}^{6}$, es un cuestionario de resultados informado por pacientes conformado por 12 ítems, agrupados en 3 subescalas, las cuales evalúan diferentes esferas de la enfermedad: síntomas oculares (ítems 1,2,3), calidad de vida relacionada a función visual (ítems 4-9) y gatillantes ambientales (ítems 10-12). En la literatura se reporta la validez, confiabilidad y reproducibilidad del $\mathrm{OSDI}^{7}$, siendo su objetivo facilitar el diagnóstico del SOS y proporcionar evidencia del deterioro en la calidad de vida ${ }^{8}$.

Con el objetivo de diagnosticar enfermedad, evaluar intensidad y monitorear respuesta terapéutica, resulta esencial el uso de cuestionarios adaptados y validados culturalmente para una población específica. Una revisión de Guillemin y cols. ${ }^{9}$ respalda la importancia de utilizar cuestionarios validados para una correcta evaluación de los síntomas de la ESO, premisa que es reforzada por los últimos reportes del DEWS II $^{4,5}$. En este contexto, el OSDI ha sido traducido y validado en francés, alemán, sueco, inglés-británico ${ }^{10}$, portugués-brasileño ${ }^{11}$, español-colombiano ${ }^{12}$, farsi-iraní1 ${ }^{13}$ y numerosos estudios han utilizado este cuestionario para la evaluación clínica del SOS $^{8,14-16}$. Actualmente existen 25 versiones traducidas del cuestionario OSDI, pero no todas ellas han sido validadas ${ }^{17}$.

Para validar un cuestionario, primero se debe adaptar culturalmente al medio donde se quiere utilizar y, a continuación, medir sus características psicométricas. Mientras más veces se hayan medido en distintas culturas, más consistente será este proceso ${ }^{18}$. Existen diversos métodos de validación de cuestionarios clínicos, pero no está reportada la superioridad de uno en particular. Una revisión de Epstein y cols. ${ }^{19}$ identifica 31 guías metodológicas, sin encontrar consenso entre ellas. Sin embargo, las principales guías recomiendan una serie de pasos bien definidos, a saber: traducción, síntesis o reconciliación de las traducciones, retrotraducción, revisión por comité de expertos y presentación ${ }^{20}$.

El objetivo de este estudio es presentar un proceso de validación lingüística y cultural del cuestionario OSDI para la población chilena en estudio, con el fin de proveer un instrumento adaptado, validado y adecuado para evaluar la ESO en la práctica clínica y estudios de investigación.

\section{Materiales y Métodos}

Se diseñó un estudio transversal, retrospectivo y descriptivo en la Unidad de Lágrima y Superficie Ocular (ULSO) del Centro de la Visión (CEV) filial Clínica Las Condes en la ciudad de Santiago de Chile, con el objetivo de adaptar culturalmente y validar el cuestionario OSDI. Se procedió a la adaptación cultural y lingüística del cuestionario desde su versión original en inglés, mediante un proceso de pasos estandarizados, generándose una versión para ser usada en la población en estudio, la cual fue validada mediante análisis psicométrico de consistencia interna y validez de constructo.

\section{Sujetos de estudio}

\section{Universo}

Entre septiembre de 2017 y agosto de 2018 se encuestaron 314 pacientes con diagnóstico de SOS según criterios del DEWS II que incorporan: preguntas y análisis de factores de riesgo. Luego se incluyen tests diagnósticos, como cuestionarios de sintomatología (OSDI y DEQ-5) y marcadores de homeostasis (BUT, osmolaridad y tinciones). Finalmente, si el paciente presenta síntomas de SOS, más, al menos un signo positivo entre los marcadores homeostáticos, se diagnóstica la enfermedad.

\section{Muestra}

Para el cálculo del tamaño de la muestra se consideró una prevalencia de SOS de $13 \%{ }^{4}$, nivel de confianza de $95 \%$ y un error de $5 \%$, quedando constituida por 200 encuestas.

Se realizó un muestreo no probabilístico consecutivo de las encuestas realizadas, las que constituyeron una base de datos secundaria. Solo se incluyeron aquellas encuestas que contaban con el mismo número de categorías de respuesta, requerimiento obligatorio para el proceso de análisis factorial mediante el programa FACTOR, y que contaron con toda la información para las variables seleccionadas en el estudio.

\section{Consideraciones éticas}

Este estudio fue revisado y aprobado por el Comité de Ética del CEV siguiendo las directrices establecidas en la declaración de Helsinki. 


\section{Adaptación lingüistica y cultural del cuestiona- rio OSDI}

La Figura 1 resume el proceso metodológico de adaptación lingüístico-cultural y la validación del cuestionario ${ }^{11,20}$. La versión final del mismo en español-chileno se muestra en la Figura 2.

\section{Análisis estadístico}

Se realizó un análisis exploratorio de los datos para verificar validez y consistencia de las variables de interés, identificando datos perdidos y outlier. Posteriormente, se obtuvieron las medias y desviaciones o medianas y rangos intercuartílicos para

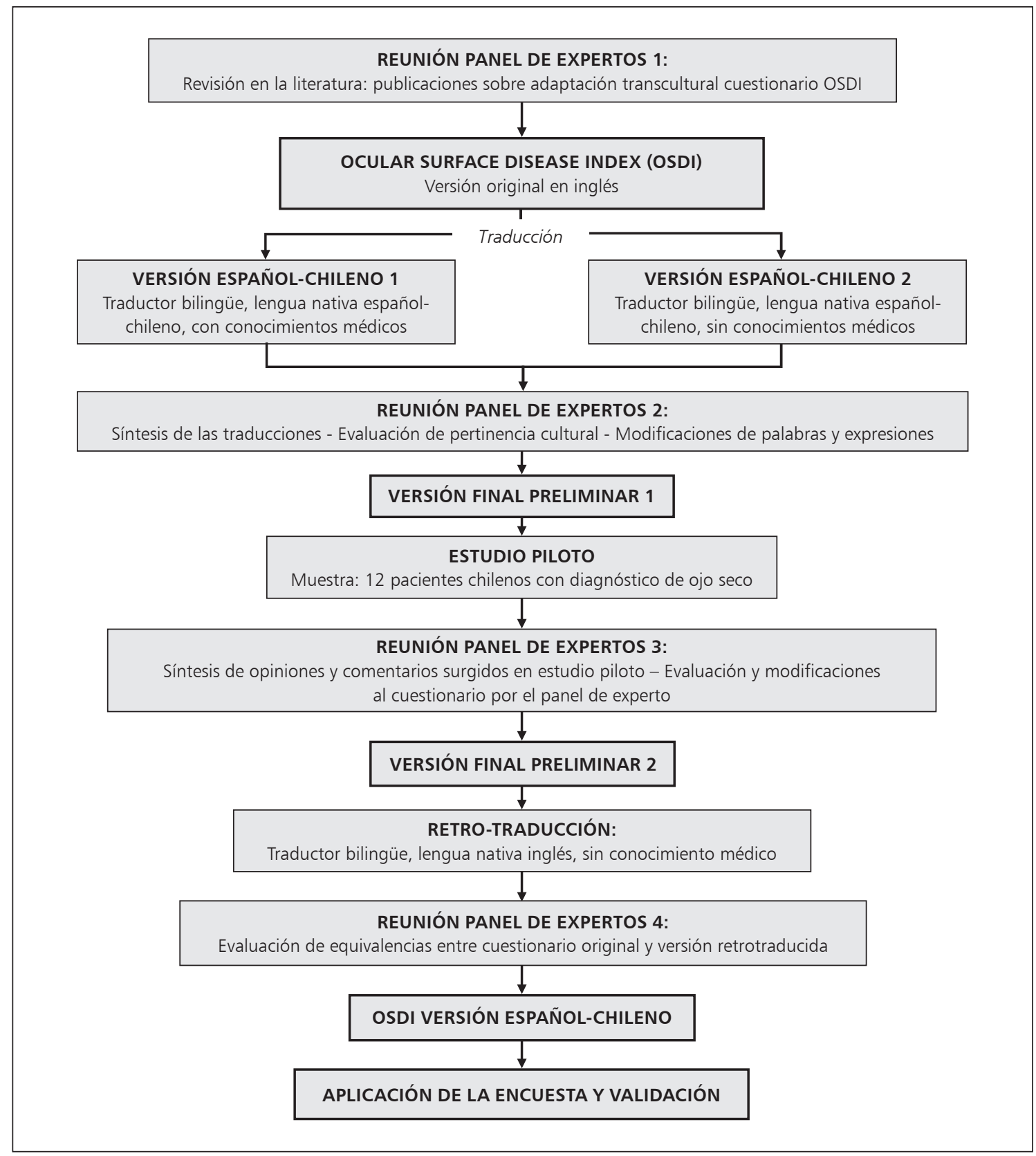

Figura 1. Metodología del estudio. 


\section{ANTECEDENTES PERSONALES}

\begin{tabular}{|l|l|}
\hline Nombre: & \\
\hline Edad : & Fecha: \\
\hline
\end{tabular}

1. ¿Ha experimentado cualquiera de los siguientes síntomas durante la última semana?

\begin{tabular}{|l|c|c|c|c|c|}
\hline & $\begin{array}{c}\text { Todo el } \\
\text { tiempo }\end{array}$ & $\begin{array}{c}\text { La mayor parte } \\
\text { del tiempo }\end{array}$ & $\begin{array}{c}\text { La mitad del } \\
\text { tiempo }\end{array}$ & $\begin{array}{c}\text { Algunas } \\
\text { veces }\end{array}$ & Nunca \\
\hline 1. Ojos sensibles a la luz & 4 & 3 & 2 & 1 & 0 \\
\hline 2. Sensación de arenilla en los ojos & 4 & 3 & 2 & 1 & 0 \\
\hline 3. Dolor en los ojos & 4 & 3 & 2 & 1 & 0 \\
\hline 4. Visión borrosa & 4 & 3 & 2 & 1 & 0 \\
\hline 5. Mala visión o visión pobre & 4 & 3 & 2 & 1 & 0 \\
\hline
\end{tabular}

Subtotal pregunta 1

2. ¿Sus problemas oculares han limitado la realización de alguna de las siguientes actividades durante la última semana?

\begin{tabular}{|l|c|c|c|c|c|c|}
\hline & $\begin{array}{c}\text { Todo el } \\
\text { tiempo }\end{array}$ & $\begin{array}{c}\text { La mayor parte } \\
\text { del tiempo }\end{array}$ & $\begin{array}{c}\text { La mitad del } \\
\text { tiempo }\end{array}$ & $\begin{array}{c}\text { Algunas } \\
\text { veces }\end{array}$ & Nunca & No aplicable \\
\hline 1. Leer o ver de cerca & 4 & 3 & 2 & 1 & 0 & 0 \\
\hline 2. Manejar de noche & 4 & 3 & 2 & 1 & 0 & 0 \\
\hline $\begin{array}{l}\text { 3. Usar computador o } \\
\text { cajero automático }\end{array}$ & 4 & 3 & 2 & 1 & 0 & 0 \\
\hline 4. Ver televisión & 4 & 3 & 2 & 1 & 0 & 0 \\
\hline
\end{tabular}

Subtotal pregunta 2

3. ¿Ha sentido molestias oculares en alguna de estas situaciones durante la última semana?

\begin{tabular}{|l|c|c|c|c|c|c|}
\hline & $\begin{array}{c}\text { Todo el } \\
\text { tiempo }\end{array}$ & $\begin{array}{c}\text { La mayor } \\
\text { parte del } \\
\text { tiempo }\end{array}$ & $\begin{array}{c}\text { La mitad } \\
\text { del tiempo }\end{array}$ & $\begin{array}{c}\text { Algunas } \\
\text { veces }\end{array}$ & Nunca & $\begin{array}{c}\text { No } \\
\text { aplicable }\end{array}$ \\
\hline 1. Lugares con viento & 4 & 3 & 2 & 1 & 0 & 0 \\
\hline 2. Lugares muy secos & 4 & 3 & 2 & 1 & 0 & 0 \\
\hline 3. Lugares con aire acondicionado & 4 & 3 & 2 & 1 & 0 & 0 \\
\hline
\end{tabular}

Subtotal pregunta 3

\begin{tabular}{|l|l|}
\hline Suma subtotales & \\
\hline Preguntas contestadas & \\
\hline $\begin{array}{l}\text { Puntaje OSDI } \\
\text { Puntaje total } \times 25 \text { /número de preguntas }\end{array}$ & \\
\hline Clasificación OSDI & \\
\hline
\end{tabular}

\begin{tabular}{|l|l|}
\hline \multicolumn{2}{|l|}{ Clasificación OSDI } \\
\hline Normal & $0-12$ puntos \\
\hline Leve & $13-22$ puntos \\
\hline Moderado & $23-32$ puntos \\
\hline Severo & $33-100$ puntos \\
\hline
\end{tabular}

Figura 2. Cuestionario OSDI final traducido, adaptado y validado para el segmento de población chilena valorado en este estudio. 
las variables cuantitativas dependiendo de la distribución de cada variable, mientras que para las variables cualitativas se calcularon las frecuencias absolutas con sus respectivas frecuencias relativas como análisis univariado para caracterizar la muestra.

\section{Evaluación psicométrica: consistencia interna y validez del constructo}

La consistencia interna se evaluó mediante estimación del coeficiente alfa de Cronbach, el cual fue cuantificado para cada ítem y la totalidad del cuestionario con un rango de 0 a 1 . La selección de ítems consideró aquellos que presentaron una correlación ítem-total mayor o igual a 0,4 , sin aumentar el estadístico alfa Cronbach al ser eliminados ítems del cuestionario con correlaciones inferiores.

Para establecer la validez de constructo, se realizó un análisis factorial con el método factorización ULS (mínimos cuadrados no ponderados). Se estableció como punto de corte para su inclusión, valores propios (eigenvalue) mayores o iguales a uno, seleccionando aquellos que presentaban cargas mayores a $0,4^{22}$.

El análisis estadístico fue realizado mediante el software Stata 13.0 (Windows. College Station, TX, USA). Se utilizó además el programa FACTOR, versión 10.8.02 (Rovira i Virgili University, Tarragona, España $)^{22}$.

\section{Resultados}

La caracterización de los encuestados se encuentra en la Tabla 1. El $81 \%$ presentó SOS (puntaje OSDI $\geq 13$ ) y, de estos, $55 \%$ en grado grave (puntaje OSDI $\geq 33$ ).

$\mathrm{Al}$ explorar la correlación de cada ítem con la escala completa (correlación ítem-test), esta fue superior a 0,4 en todas las preguntas, por lo que no fue necesario eliminar ninguna de ellas. El coeficiente alfa de Cronbach alcanzó un valor de 0,91 , considerado como una "excelente" consistencia interna. Los valores del coeficiente alfa de Cronbach del total del cuestionario se muestran en la Tabla 2.

Del análisis factorial, se estableció la predominancia de 3 factores responsables de explicar $75,4 \%$ de la varianza total. De acuerdo a su estructura, los factores fueron denominados como:
"Ambiental", "Estilos de vida" e "Individual/ síntomas" (Tabla 3 ).

Los tres factores agruparon los ítems o preguntas del cuestionario adaptado lingüísticamente en este estudio coincidiendo en la mayoría de los casos con la agrupación del cuestionario original. Sin embargo, el Factor 1 se diferenció en la incorporación de la pregunta: “¿sensación de arenilla en los ojos?", la que en el cuestionario original se agrupó junto a preguntas rotuladas bajo el constructo síntomas. En el Factor 2, que agrupó ítems que hacían mención a actividades o situaciones específicas de la vida cotidiana, se agregó el ítem: "lugares con viento", el cual se incluye en el constructo de "gatillantes ambientales" del cuestionario original. El factor 3 presentó la menor variabilidad y agrupó los ítems como el cuestionario original, a excepción de la pregunta 2 (Tabla 4).

Tabla 1. Características de la muestra

\begin{tabular}{|lrc|}
\hline Característica & $\mathbf{n}$ & $\%$ \\
Sexo & & \\
Mujer & 147 & 73,50 \\
Hombre & 53 & 26,50 \\
Edad & & \\
$0-20$ & 5 & 2,5 \\
$21-30$ & 21 & 10,5 \\
$31-40$ & 26 & 13 \\
$41-50$ & 27 & 13,5 \\
$51-60$ & 45 & 22,5 \\
$61-70$ & 47 & 23,5 \\
$71-80$ & 25 & 12,5 \\
81 y más & 4 & 2,0 \\
Sin ojo seco & 38 & 19,00 \\
Con ojo seco & 162 & 81,00 \\
Leve & 25 & 12,50 \\
Moderado & 27 & 13,50 \\
Grave & 110 & 55,00 \\
\hline
\end{tabular}

Se presenta la distribución por sexo y edad para la muestra de 200 encuestados. Además, se observa el valor absoluto y relativo de pacientes catalogados como ojo seco ( $>12$ puntos) y su nivel de gravedad (Leve = 13-22 puntos; Moderado= 23-32 puntos; Grave $\geq 33$ puntos) según puntaje obtenido en la versión final del OSDI resultante de este estudio. 
Tabla 2. Evaluación de la confiabilidad para cada ítem del cuestionario OSDI

\begin{tabular}{|lcc|}
\hline Pregunta & Correlación ítem total & Alfa de Cronbach \\
\hline ¿Ojos sensibles a la luz? & 0,6671 & 0,9039 \\
\hline ¿Sensación de arenilla en los ojos? & 0,5987 & 0,9075 \\
\hline ¿Dolor en los ojos? & 0,5905 & 0,9080 \\
¿Visión borrosa? & 0,6930 & 0,9025 \\
\hline ¿Mala visión? & 0,6108 & 0,9069 \\
\hline ¿Dificultad para leer? & 0,7517 & 0,8992 \\
\hline ¿Dificultad para manejar de noche? & 0,7251 & 0,9007 \\
\hline ¿Dificultad para usar computador o cajero automático? & 0,8966 \\
\hline ¿Dificultad para ver televisión? & 0,7243 & 0,9008 \\
\hline ¿Molestias en lugares con viento? & 0,7897 & 0,8053 \\
\hline ¿Molestias en lugares muy secos? & 0,61122 & 0,8971 \\
\hline ¿Molestias en lugares con aire acondicionado? & 0,7318 & 0,9004 \\
\hline Total & & 0,9092 \\
\hline
\end{tabular}

Se evaluó la correlación ítem total y el alfa de Cronbach para cada uno de los ítems del cuestionario. El valor del alfa de Cronbach para el cuestionario en general fue de 0,9092, lo cual califica como "excelente".

Tabla 3. Factores retenidos de análisis factorial mediante rotación Quartimin Normalizado

\begin{tabular}{|llccc|}
\hline Factor & \multicolumn{1}{c}{ Categoría } & $\begin{array}{c}\text { Varianza } \\
\text { Eigenvalue }\end{array}$ & $\begin{array}{c}\text { Proporción de varianza } \\
\text { total }\end{array}$ & $\begin{array}{c}\text { Proporción de varianza } \\
\text { total acumulada }\end{array}$ \\
\hline Factor 1 & Ambiental & 6,636 & 0,553 & 0,553 \\
\hline Factor 2 & Estilos de vida & 1,326 & 0,110 & 0,663 \\
\hline Factor 3 & Individual/síntomas & 1,080 & 0,090 & 0,753 \\
\hline
\end{tabular}

Se presentan los 3 factores que representan el 75,4\% de la varianza en las respuestas de los encuestados. Para cada factor se observa el valor absoluto de la varianza y la proporción de esta sobre la varianza total. De acuerdo a los ítems que agrupó cada factor, se les otorgó una denominación específica en la columna de "categoría" que indica la esfera de la sintomatología de ojo seco que se evalúa.

Tabla 4. Estructura de factores retenidos rotados por método de Quartimin Normalizado

\begin{tabular}{|lll|}
\hline Categoría & Factor $\mathbf{1}$ & Factor 2 \\
\hline Ambiental & & \\
$\quad$ ¿Molestias en lugares muy secos? & 0,841 & \\
¿Molestias en lugares con aire acondicionado? & 0,857 & \\
¿Sensación de arenilla en los ojos? & 0,878 & \\
Estilos de vida & & \\
¿Dificultad para leer? & & 0,602 \\
¿Dificultad para manejar de noche? & 0,706 \\
¿Dificultad para usar computador o cajero automático? & 0,675 \\
¿Dificultad para ver televisión? & 0,775 \\
¿Molestias en lugares con viento? & 0,817 \\
Individual/síntomas & \\
$\quad$ ¿Ojos sensibles a la luz? & \\
¿Dolor en los ojos? & \\
¿Visión borrosa? & & \\
¿Mala visión? & & 0,654 \\
\hline
\end{tabular}

Se observan cada uno de los ítems agrupados de acuerdo al factor. 


\section{Discusión}

Con respecto a la validación del cuestionario OSDI, la consistencia interna calificó cualitativamente como excelente, tanto en la homogeneidad de sus enunciados como en la relación entre ellos. A través del análisis factorial, los ítems se agruparon en tres factores que explicaron $75,4 \%$ de la variabilidad total.

La mayor parte de los sujetos con SOS fueron de sexo femenino $(73,5 \%)$, aumentando esta proporción a mayor edad. Estos resultados se corresponden con la epidemiología del SOS y otros estudios ${ }^{12}$ que evidencian, como factores de riesgo, la edad y el sexo femenino asociado, principalmente, a la caída de andrógenos producto de la menopausia. En este estudio hay una alta proporción de pacientes con SOS grave (puntaje OSDI $\geq 33$ ), lo cual puede deberse a que en ULSO, las molestias por ojo seco son un motivo habitual de consulta.

A pesar que los sujetos de la muestra tenían previamente diagnóstico de SOS, 19\% presentó puntaje OSDI normal. Esto podría explicarse porque el cuestionario pregunta por molestias en la última semana y la sintomatología de los pacientes con SOS es variable, además de la influencia del efecto memoria.

Una publicación que valida el OSDI al portugués-brasileño ${ }^{11}$ propone un esquema metodológico similar a este estudio, pero no incorpora una fase piloto, sino que, en su lugar, 2 estrategias secuenciales: grupos focales y entrevistas intelectuales. Además, tanto el presente estudio como el de Santos M. ${ }^{11}$ contaron con 2 traductores bilingües con lengua nativa, correspondiente al de la población objetivo. No hubo participación de un lingüista. Sin embargo, para la retrotraducción el artículo de Santos M. ${ }^{11}$ posee 2 traductores, mientras que el presente estudio solo uno. Aunque la retrotraducción es una fase recomendada, su aporte en el instrumento resultante no ha sido demostrado ${ }^{23}$, ergo, el número de retrotraductores no debiera generar diferencias. Puesto que ambos estudios muestran resultados similares, estas diferencias metodológicas no tienen aparentemente un impacto significativo.

El estudio de Schiffman y cols. ${ }^{7}$ evaluó la confiabilidad y validez del cuestionario OSDI original en una muestra de 109 pacientes con SOS y 30 controles normales, reportando un análisis de fia- bilidad general del test de 0,92 , similar al obtenido en este estudio, de 0,91. Al evaluar la fiabilidad específica de las 3 subescalas (funcionalidad relacionada a visión, síntomas oculares y gatillantes ambientales), Schiffman y cols. ${ }^{7}$ reportan valores de alfa de Cronbach de 0,88; 0,92 y 0,78, respectivamente, mientras que en el presente estudio se hallaron cifras de 0,$77 ; 0,80$ y 0,80 , respectivamente. En ambos casos los resultados son interpretados en un rango de bueno a excelente.

El alfa de Cronbach alcanzó un valor de 0,91 en este estudio y de 0,$905 ; 0,9$ y 0,87 para la validación del OSDI al portugués-brasileño ${ }^{11}$, español-colombiano ${ }^{12}$ y farsi-iraní ${ }^{13}$, respectivamente, cifras cercanas al 0,92 reportada para el OSDI original ${ }^{7}$.

Existen otras publicaciones que apuntan a adecuar el test en su medio cultural. Un estudio de Beltran F. y cols reporta la adaptación cultural del OSDI para población mexicana, pero no su validación ${ }^{24}$. Por otro lado, una versión de OSDI en turco obtuvo cifras de sensibilidad y especificidad de $100 \%$ y $67 \%$, respectivamente y no detectó diferencias de un mismo grupo de pacientes sometidos al test en 2 tiempos $(\mathrm{p}<0,05)$. Sin embargo, no se realizó un análisis de validez de constructo como en este estudio ${ }^{25}$.

El análisis factorial realizado en este estudio agrupó algunas preguntas o ítems en dominios diferentes al del OSDI original. La pregunta número 2 (“ ¿sensación de arenilla en los ojos?”) se agrupó en el factor 1 denominado "Ambiental". Sin embargo, en el cuestionario original se agrupa junto a preguntas rotuladas como "Síntomas". Esta diferencia podría explicarse porque predominó este síntoma al estar en lugares secos o con aire acondicionado, a diferencia de los otros síntomas enumerados y lugares en que se encontraban (Tabla 5).

Por otro parte, la pregunta número 10 (“¿ilugares con viento?") se agrupó en el factor 2 denominado "Estilos de vida", aunque en el cuestionario original se ubica en "Gatillantes ambientales". Esto podría explicarse porque las personas realizan estas actividades en lugares cerrados y climatizados, lo que podría homologarse a lugares con vientos (Tabla 5).

En el factor 3 se agruparon los síntomas "ojos sensibles a la luz", "dolor en los ojos", "visión borrosa" y "mala visión", debido a que estos están presentes en los encuestados, independiente de la actividad que realicen o lugar donde se encuen- 
Tabla 5. Distribución de las preguntas del cuestionario en los 3 factores obtenidos a partir del análisis factorial de la versión del OSDI resultante de este estudio y su comparación con la versión original del OSDI

\begin{tabular}{|c|c|c|c|}
\hline & OSDI versión español- chileno & & OSDI original \\
\hline Factor & Agrupación de preguntas & Constructo & Constructo y preguntas \\
\hline Factor 1 & $\begin{array}{l}\text { P11: Molestias en lugares muy secos } \\
\text { P12: Molestias en lugares con aire acondicionado } \\
\text { P2: Sensación de arenilla en los ojos }\end{array}$ & Ambiental & $\begin{array}{l}\text { Gatillantes ambientales } \\
\text { Preguntas: } 10,11 \text {, y } 12\end{array}$ \\
\hline Factor 2 & $\begin{array}{l}\text { P6: Dificultad para leer } \\
\text { P7: Dificultad para manejar de noche } \\
\text { P8: Dificultad para usar computador o cajero automático } \\
\text { P9: Dificultad para ver televisión } \\
\text { P10: Molestias en lugares con viento }\end{array}$ & Estilos de vida & $\begin{array}{l}\text { Calidad de vida relacionada } \\
\text { a función visual } \\
\text { Preguntas: 6-9 }\end{array}$ \\
\hline Factor 3 & $\begin{array}{l}\text { P1: Ojos sensibles a la luz } \\
\text { P3: Dolor en los ojos } \\
\text { P4: Visión borrosa } \\
\text { P5: Mala visión }\end{array}$ & Individual/síntomas & $\begin{array}{l}\text { Síntomas } \\
\text { Preguntas: } 1-5\end{array}$ \\
\hline
\end{tabular}

$\mathrm{PX}=$ pregunta del cuestionario OSDI original, donde " $\mathrm{X}$ " es el número específico de la pregunta.

tren. Tales síntomas podrían representar mejor, para la población de este estudio, la sintomatología de ojo seco que la agrupación de síntomas del cuestionario original (Tabla 5).

El dominio de "Gatillantes ambientales" pregunta por condiciones ambientales y actividades de la vida diaria que desencadenan o empeoran la sintomatología. La realidad socioeconómica, educacional y ambiental podría determinar experiencias y acciones cotidianas que son preguntadas en el OSDI. En este estudio, dichas preguntas presentaron buena consistencia interna (alfa de Cronbach mayor a 0,89). Esto refleja que la mayoría de los encuestados reconoce y realiza estas acciones en su vida cotidiana.

Si bien no es posible extrapolar estos resultados para toda la población chilena, esta es la primera y única versión del cuestionario OSDI adaptada culturalmente y validada para un segmento de dicha población.

Para concluir, un proceso de adaptación cultural y análisis psicométrico, estandarizado según las recomendaciones metodológicas actuales, permite presentar una versión del cuestionario OSDI culturalmente adaptada y validada para un segmento de población chilena y probar que es un instrumento válido y confiable para evaluar el SOS.

Para futuras investigaciones que incorporen la adaptación cultural y lingüística de cuestionarios, sería recomendable integrar profesionales expertos en lingüística, para cautelar el uso del lenguaje más representativo de la población en estudio y la versión adaptada más cercana al cuestionario de origen.

\section{Referencias}

1. Begley CG, Chalmers RL, Abetz L, Venkataraman K, Mertzanis P, Caffery B, et al. The Relationship between Habitual Patient-Reported Symptoms and Clinical Signs among Patients with Dry Eye of Varying Severity. Investig Ophthalmol Vis Sci 2003; 44 (11): 4753-61.

2. Nichols KK, Nichols JJ, Mitchell GL. The lack of association between signs and symptoms in patients with dry eye disease. Cornea 2004; 23 (8): 762-70.

3. Fuentes-Páez G, Herreras JM, Cordero Y, Almaraz A, González M, Calonge M. Falta de concordancia entre los cuestionarios y las pruebas diagnósticas en el síndrome de ojo seco. Arch Soc Esp Oftalmol 2011; 86 (1): 3-7.

4. Stapleton F, Alves M, Bunya VY, Jalbert I, Lekhanont $\mathrm{K}$, Malet F, et al. TFOS DEWS II Epidemiology Report. Ocul Surf 2017; 15 (3): 334-65.

5. Wolffsohn JS, Arita R, Chalmers R, Djalilian A, Dogru $\mathrm{M}$, Dumbleton K, et al. TFOS DEWS II Diagnostic Methodology report. Ocul Surf 2017; 15 (3): 539-74.

6. Walt J, Rowe M, Stern K. Evaluating the functional Impact of Dry Eye: The Ocular Surface Disease Index. Drug Inf J 1997; 31: 1436. 
7. Schiffman RM, Christianson MD, Jacobsen G, Hirsch JD, Reis BL. Reability and validity of the Ocular Surface Disease Index. Arch Ophthalmol 2000; 118 (5): 615-21.

8. Miller KL, Walt JG, Mink DR, Satram-Hoang S, Wilson S, Perry H, et al. Minimal Clinically Important Difference for the Ocular Surface Disease Index. Arch Ophthalmol 2010; 128 (1): 94-101.

9. Guillemin ISG, Begley CAB, Chalmers ROC, Baudouin CHB, Arnould BEA. Appraisal of Patient-Reported Outcome Instruments Available for Randomized Clinical Trials in Dry Eye : Revisiting the Standards. Ocul Surf 2012; 10 (2): 84-99.

10. Santo RM, Ribeiro-Ferreiro F, Alves MR, Epstein J, Novaes P. Enhancing the cross-cultural adaptation and validation process: Linguistic and psychometric testing od thr Brazilian Portugese version of a self-report measure for dry eye. J Clin Epidemiol 2015; 68 (4): 370-8.

11. Santo RM, Ribeiro-Ferreira F, Alves MR, Epstein J, Novaes P. Enhancing the cross-cultural adaptation and validation process: Linguistic and psychometric testing of the Brazilian-Portuguese version of a self-report measure for dry eye. J Clin Epidemiol 2015; 68 (4): 370-8.

12. González J, Ulloa I, Correa O, Prieto L. Reliability and Validity of the Questionnaire OSDI (Ocular Surface Disease Index) in Patients Diagnosed with Dry Eye Syndrome in the Hospital Simon Bolivar, Colombia Fiabilidad y Validez del Cuestionario OSDI (Ocular Disease Surface Index) en Pacient. Rev Soc Colomb Oftalmol 2015; 48 (3): 262-76.

13. Pakdel F, Gohari MR, Jazayeri AS, Amani A. Validation of Farsi Translation of the Ocular Surface Disease Index. J Ophthalmic Vis Res 2017; 12 (3): 301-4.

14. Skalicky SE, Goldberg I, Mccluskey P. Ocular Surface Disease and Quality of Life in Patients With Glaucoma. AJOPHT 2012; 153 (1): 1-9.e2.

15. Vandermeid KR, Su SP, Ward KW, Zhang J. Correlation of Tear Inflammatory Cytokines and Matrix Metalloproteinases with Four Dry Eye Diagnostic Tests 2012; 53 (3): 1512-8.

16. Jacobi C, Bellios N, Jacobi A, Kruse F, Cursiefen C.
Erhebungsbogen zur Anamnese und Diagnostik des trockenen Auges Screening Questionnaire for Documentation of Medical History and Diagnostic Findings in Dry Eye Disease. Klin Monbl Augenheilkd 2011; 228 (3): 226-23.

17. Ocular Surface Disease Index. Versiones traducidas. Disponible en: https://eprovide.mapi-trust.org/instruments/ocular-surface-disease-index [Consultado el 19 de mayo de 2019].

18. Tsang S, Royse CF, Terkawi AS. Guidelines for developing, translating, and validating a questionnaire in perioperative and pain medicine. Saudi J Anaesth 2017; 11 (Suppl 1): S80-9.

19. Epstein J, Santo RM, Guillemin F. A review of guidelines for cross-cultural adaptation of questionnaires could not bring out a consensus. J Clin Epidemiol 2015; 68 (4): 435-41.

20. Beaton D, Bombardier C, Guillemin F, Ferraz M. Guidelines for the process of cross-cultural adaptation of self-report measures. Spine (Phila Pa 1976) 2000; 25 (24): 3186-91.

21. Guillemin F, Bombardier D, Bearton D. Cross-cultural adaptation of health-related quality of life measures: literature review and proposed guidelines. J Clin Epidemiol 1993; 46 (12): 1417-32.

22. Lloret-Segura S, Ferreres-Traver A, Tomás-Marco AHI. El análisis factorial exploratorio de los ítems : una guía práctica, revisada y actualizada. An Psicol 2014; 30 (3): 1151-69.

23. Perneger TV, Leplège A, Etter J-F. Cross-Cultural Adaptation of a Psychometric Instrument. J Clin Epidemiol 1999; 52 (11): 1037-46.

24. Beltrán F, Ramos Betancourt N, Martínez J, et al. Mexican OSDI Study Group. Transcultural Validation of Ocular Surface Disease Index (OSDI) Questionnaire for Mexican Population. Invest Ophthalmol Vis Sci 2013; 54: 6050.

25. Irkec MT. Turkish OSDI Study Group. Reliability and Validity of Turkish Translation of the Ocular Surface Disease Index (OSDI) in Dry Eye Syndrome. Invest Ophthalmol Vis Sci 2007; 40: 408. 